Euskal ikerketen aldizkaria | Revue d'études basques |

Revista de estudios vascos | Basque studies review

$15 \mid 2011$

Numéro XV

\title{
Professeur Pierre Bidart
}

Jean-Baptiste Orpustan

URL : http://journals.openedition.org/lapurdum/2337

DOI : 10.4000/lapurdum.2337

ISSN : 1965-0655

Éditeur

IKER

Édition imprimée

Date de publication : 1 octobre 2011

Pagination : 149-153

ISSN : 1273-3830

Référence électronique

Jean-Baptiste Orpustan, « Professeur Pierre Bidart », Lapurdum [En ligne], 15 | 2011, mis en ligne le 01 novembre 2014, consulté le 04 mai 2019. URL : http://journals.openedition.org/lapurdum/2337 ; DOI 10.4000/lapurdum.2337 


\title{
In memoriam : Profesor Pierre Bidart
}

\author{
Jean-Baptiste ORPUSTAN \\ IKER (UMR 5478) \& Université Michel de Montaigne - Bordeaux 3
}

La nouvelle a été aussi brutale qu'inattendue: Pierre Bidart professeur en anthropologie sociale et ethnologie à l'Université Victor Segalen-Bordeaux 2, nommé durant l'été en mission pour deux ans comme attache de coopération universitaire à l'Institut français de Sofia auprès de l'ambassade de France en Bulgarie, nous a quittés. Dans les jours qui ont suivi son décès à Sofia dans la nuit du jeudi 16 au vendredi 17 septembre, la presse de tous horizons a rappelé au public l'importance et la diversité de ses publications et de ses travaux, au cours d'un périple universitaire bien fourni, qui le mena de l'Université de Metz où débuta sa carrière, à celle de Pau et Pays de 1'Adour et la Faculté de Bayonne, puis à l'Université Victor SegalenBordeaux 2. Il faut y ajouter les nombreuses collaborations avec des universités étrangères, de l'Afrique du Nord à l'Europe centrale.

Mais c'est dans le pays et la société basques que se trouvaient le point fixe et le centre vivant d'où émanait et où retournait avec constance l'activité de recherche de Pierre Bidart sur la société et les groupes humains. C'est là que ses ouvrages principaux ont pris le plus souvent leurs objets d'analyse et de réflexion, publiés en nombreux articles, actes de colloques (il en a organisé lui-même un grand nombre aux thèmes variés) et de conférences, et des ouvrages parmi lesquels La singularité basque (PUF Ethnologies, collection dirigée par Jean Cuisenier, Paris 2001) issu de sa thèse de Doctorat d'Etat reste, dans la perspective personnelle choisie par l'auteur, un monument d'analyse anthropologique. Le « pays » de Pierre Bidart porte un nom particulier et une situation bien précise: Saint-Etienne-de-Baïgorry et la maison familiale au quartier d'Occos, et le siège du Comité Izpegi qu'il y a créé de toutes pièces et installé au centre de la commune. A peu de distance de là, dans le périmètre de l'historique BasseNavarre, la rénovation de la maison hospitalière d'Irissarry, Irisarri-Ospitalea, acquise par le Conseil Général des Pyrénées-Atlantiques, lui offrait l'espace pour organiser depuis quelques années des journées d'Université d'été de Bordeaux 2.

Pierre Bidart a tenu un rôle, par moments discret, mais de premier plan dans la mise en place et le développement des études et des enseignements universitaires, et en même temps des publications de langue, littérature et culture basques, et c'est plus particulièrement à ce titre que l'auteur de ces lignes et la revue Lapurdum lui doivent une reconnaissance et un hommage auxquels s'associent tous ceux de ses amis, collègues, et étudiants qui en ont bénéficié.

Les études universitaires basques en France, jusque-là représentées par l'unique chaire d'Etudes Basques que tenait le Professeur Jean Haristchelhar à 1'Université de Bordeaux 3, qui venait de créer un Institut Universitaire d'Etudes Basques dans la Faculté de Droit et de Sciences Economiques de Bayonne, bientôt suivi à Bordeaux III d'un centre de recherches associé au CNRS, a pris un nouveau tournant à partir de 1981, avec la création de deux postes d'assistant en études basques: l'un à Bordeaux 3, l'autre à Pau. Ce fut justement Pierre Bidart qui m'apprit au téléphone cette double création; elle allait permettre un nouveau développement des enseignements universitaires de basque, et l'obtention, non sans difficultés dues à l'extrême réticence de l'Administration et pour remplacer les diplômes d'Université délivrés à Bayonne 
par Bordeaux 3, les diplômes d'Etat. Nous étions réunis pour un colloque organisé par Pierre Bidart à Baïgorry dans l'été 1989, quand le Préfet de l'époque nous annonça, après bien des démarches et dossiers adressés au ministère depuis plusieurs années, que la décision de créer un DEUG était enfin prise en « haut lieu ».

Le cadre administratif universitaire et l'instance qui organiseraient et délivreraient ces diplômes restaient à établir, pour un centre d'enseignement jusque-là assuré à Bayonne par Bordeaux III dans des locaux et un site dépendant de celle de Pau et Pays de l'Adour. Le résultat, négocié un peu laborieusement il est vrai, a été ce « Département Interuniversitaire d'Etudes Basques » intégré dans la Faculté pluridisciplinaire de Bayonne, de ce fait administrativement rattaché à Pau, dont les enseignements sont assures conjointement par les enseignantschercheurs des deux Universités et les diplômes délivrés sous leur double sceau. Les diplômes de second cycle, licence et maîtrise, sont venus y compléter le cycle universitaire des études basques auxquelles Pierre Bidart a participé pendant plusieurs années pour sa discipline. Le CAPES enfin en 1993 mettait en place la formation des enseignants du secondaire. Les enseignements du DEA d'Etudes Basques et le doctorat assures jusque-là à Bordeaux III, ainsi que le centre de recherche associé au CNRS (aujourd'hui «IKER ») avaient rejoint aussi le Département Interuniversitaire.

Pierre Bidart a d'emblée acquiescé à l'idée de créer, à côté du centre de recherches, et pour en manifester l'activité en publication régulière, une revue annuelle au nom emprunté à l'antique cité bayonnaise ou « labourdine » qui accueillait ces activités universitaires de recherches en langue et culture basques: Lapurdum. Sa contribution a été déterminante pour obtenir l'aide de l'Université de Pau à cette entreprise, trouver le premier éditeur (celui des 6 premiers numéros), bref mettre en place un outil de diffusion des recherches en langue et culture basques. Commencée au n ${ }^{\circ}$ I en 1996, elle se poursuit, en cette année 2011, avec la publication de ce numéro XV.

Au cours d'un de ces repas du dimanche en famille où il nous accueillait dans la maison Iguzkialdea du « quartier d'Occos en Baïgorry » (selon la vieille formule), à deux pas de la maison paternelle Ardantzea (il a dû être heureux de la voir rester dans sa famille), Pierre Bidart lança l'idée de créer à Saint-Etienne-de-Baïgorry une maison d'édition. Il y avait bien des maisons d'éditions localisées à bonne distance des centres parisiens et qui n'en vivaient pas moins bien leur destin d'éditeur. Le projet, pour audacieux qu'il eût pu paraître, fut immédiatement accepté, et même non sans quelque enthousiasme. On se mit à chercher le nom des éditions dans quelque toponyme local assez typique pour être significatif et porter en même temps la marque d'origine: je proposai, un peu trop facilement, «Iparla», aux crêtes fameuses; mais le nom était déjà pris. Ce fut en définitive Izpegi. Nom très bien venu en réalité, par son passé de liaison et de relations suivies, en dépit des drames historiques et politiques, du pays de Baïgorry et de la Basse-Navarre avec la vallée du Bastan et la Haute-Navarre. Il comportait en lui-même le ferment des nouvelles relations pour ainsi dire « navarro-navarraises », aux deux côtés de la frontière d'Etat, auxquelles Pierre Bidart a oeuvré ces dernières années avec tant d'efficacité, contribuant ainsi à redonner vie, dans le domaine des activités intellectuelles et scientifiques - et non plus des seuls échanges touristiques ou commerciaux, quand il ne s'agissait pas, plus discrètement, de contrebande -, à un chemin millénaire qui ne semble pas près de se refermer.

Les éditions Izpegi, fondues dans le Comité Izpegi que Pierre Bidart a dirigé depuis qu'il l'a créé, avec le concours d'un comité de lecture et de la présence diligente de Marie-Baptiste 
Etcheverry sa compagne pour la tenue des comptes, ont publié à ce jour une bonne trentaine de livres, y compris les deux numéros de la Revue d'histoire industrielle des Pyrénées occidentales. Ces ouvrages, répartis en collections thématiques, couvrent un large champ pluridisciplinaire: histoire et ethnographie régionales sous leurs divers aspects (en particulier la collection des «Pays » portant sur quatre des sept vallées ou pays de Basse-Navarre et la Soule: le coup d'essai fut comme il se doit une "Vallée de Baïgorry et ses alentours » remplacé plus tard par une "Vallée de Baïgorry » plus étoffée); langue et lettres basques, collection qui remit notamment à la portée du public un de nos « classiques », le parémiologue et poète du XVIIe siècle Arnaud d'Oyhénart; actes de colloques et thèses réunis dans la collection des « Recherches », et d'autres encore. Six des ouvrages ainsi publiés de 1990 à 2010 sont aujourd'hui épuisés, d'autres réédités, ce qui témoigne assez du besoin de culture régionale, sans aucun esprit d'exclusivité, auquel a répondu et répond encore le projet de Pierre Bidart. Il répondait en même temps et très consciemment à un besoin auquel l'édition en France n'avait guère d'outil à fournir: celui de l'enseignement et des formations universitaires en langue et culture basques qui se sont mis en place durant la même période. Champ vaste el nouveau dans notre région, auquel les Editions Izpegi ont participé de façon pionnière et décisive, et, peut-on l'espérer, sauront aussi continuer dans cette voie, à côté des nombreuses autres activités d'éducation et de formation, le CPIE en particulier, du Comité Izpegi, pour lesquelles Pierre Bidart a reçu la croix de la Légion d'Honneur. Ancien de la Casa de Velasquez, il était aussi décoré de l'ordre d'Isabelle le Catholique.

Au nom de toute l'équipe de rédaction de Lapurdum et du Centre de recherches en études basques IKER, il me revient de dire ici, avec l'expression de nos très sincères condoléances à sa famille et à ses proches dont nous partageons la peine, la gratitude et la reconnaissance à Pierre Bidart pour la part qu'il a prise dans le développement des études basques dans notre région, et de rendre hommage à sa mémoire en cette brève épitaphe (ilhartitz: un genre et un mode poétiques quelque peu « oyhénartiens »-que les Muses et les Académies pardonnent ce néologisme local -), dans le «tutoiement » basque qu'il affectionnait dans ses relations quotidiennes:

\section{Ilhartitz}

Izpegitik Lapurdi,

Bordeletik Bulgaria:

Bete duk egintzez bizia.

Euk hemen, Pierre Bidart,

Heure lagunen orhoita,

Bai et' Euskal-Herritarrena.

Jean-Baptiste ORPUSTAN, Professeur des Universités honoraire (Bordeaux III), 2010. 